\title{
Phase Diagram, Symmetry Breaking, and Critical Behavior of Three-Dimensional Lattice Multiflavor Scalar Chromodynamics
}

\author{
Claudio Bonati $\odot,{ }^{1}$ Andrea Pelissetto $\odot,{ }^{2}$ and Ettore Vicari ${ }^{1}$ \\ ${ }^{1}$ Dipartimento di Fisica dell'Università di Pisa and INFN Largo Pontecorvo 3, I-56127 Pisa, Italy \\ ${ }^{2}$ Dipartimento di Fisica dell'Università di Roma Sapienza and INFN Sezione di Roma I, I-00185 Roma, Italy
}

(Received 17 October 2019; published 4 December 2019)

\begin{abstract}
We study the nature of the phase diagram of three-dimensional lattice models in the presence of nonAbelian gauge symmetries. In particular, we consider a paradigmatic model for the Higgs mechanism, lattice scalar chromodynamics with $N_{f}$ flavors, characterized by a non-Abelian $\operatorname{SU}\left(N_{c}\right)$ gauge symmetry. For $N_{f} \geq 2$ (multiflavor case), it presents two phases separated by a transition line where a gauge-invariant order parameter condenses, being associated with the breaking of the residual global symmetry after gauging. The nature of the phase transition line is discussed within two field-theoretical approaches, the continuum scalar chromodynamics, and the Landau-Ginzburg-Wilson (LGW) $\Phi^{4}$ approach based on a gauge-invariant order parameter. Their predictions are compared with simulation results for $N_{f}=2,3$ and $N_{c}=2-4$. The LGW approach turns out to provide the correct picture of the critical behavior at the transitions between the two phases.
\end{abstract}

DOI: 10.1103/PhysRevLett.123.232002

Local gauge symmetries are key features of theories describing fundamental interactions [1] and emerging phenomena in condensed matter physics [2]. The largescale properties of three-dimensional (3D) gauge models and the nature of their thermal or quantum transitions are of interest in several physical contexts. For instance, they are relevant for superconductivity [3], topological order, and quantum transitions [4-8], and also in high-energy physics, as they describe the finite-temperature electroweak and strong-interaction transition that occurred in the early Universe [9] and which is presently being investigated in heavy-ion collisions [10].

We discuss a 3D model of interacting scalar fields with a non-Abelian gauge symmetry, which we may call scalar chromodynamics or the non-Abelian Higgs model. It provides a paradigmatic example for the non-Abelian Higgs mechanism, which is at the basis of the standard model of the fundamental interactions [11]. In condensed matter physics, it may be relevant for systems with emerging non-Abelian gauge symmetries (see, e.g., Ref. [6]). It represents the natural extension of Abelian Higgs models, which have been extensively studied in various contexts (see, e.g., Refs. [3-5,12-15]). We will focus on the multiflavor case $N_{f} \geq 2$. For $N_{f}=1$, scalar non-Abelian models have been carefully investigated, as

Published by the American Physical Society under the terms of the Creative Commons Attribution 4.0 International license. Further distribution of this work must maintain attribution to the author(s) and the published article's title, journal citation, and DOI. Funded by SCOAP. they are relevant for the finite-temperature behavior of the electroweak theory [16-20]. Much less is known about the phase diagram and the nature of the transitions (symmetrybreaking pattern, universality class, etc.) in the multiflavor case and about the effective field theory that describes the critical behavior.

In this Letter, we consider 3D lattice models of complex matrix scalar fields, with $N_{c} \times N_{f}$ components, minimally coupled to an $\mathrm{SU}\left(N_{c}\right)$ gauge field [21]. We investigate their phase diagram, for various values of $N_{f} \geq 2$ and $N_{c}$, and the nature of their phase transitions. Our numerical results allow us to understand which effective field theory provides the correct description of the phase transition. This Letter therefore provides information on the field-theoretical approach to be used to analyze thermal and quantum transitions in the presence of emergent non-Abelian gauge symmetries. Moreover, it may provide information on the finite-temperature phase diagram of non-Abelian gauge models involving scalar fields, which are meant to describe the new physics beyond the standard model of fundamental interactions.

Classical and quantum phase transitions have traditionally been studied using statistical field theories [22]. Their properties depend on the global symmetry of the model, the symmetry-breaking pattern, and some other global properties, such as the space dimensionality. In the presence of gauge symmetries, one may also think that the gaugesymmetry group is a distinctive element that should be specified to characterize the transition. As we shall discuss, however, this is not necessarily true, as gauge modes are not necessarily critical at the transition. For the same reason, at 
variance with systems that only have global symmetries, there is not a unique natural effective theory for the transition. One can, of course, consider the continuum gauge theory that corresponds to the lattice model. However, one may also consider a Landau-Ginzburg-Wilson (LGW) $\Phi^{4}$ theory based on a gauge-invariant order-parameter field with the global symmetry of the model $[15,23]$. Note that, while in the first approach the gauge symmetry is still present in the effective model, in the second one, gauge invariance does not play a particular role besides fixing the order parameter. The LGW approach is expected to be the correct one when the gauge interactions are short ranged at the transition. In the opposite case, instead, the continuum gauge model should allow for the correct picture. We recall that the LGW approach was used to predict the nature of the finite-temperature phase transition of hadronic matter in the massless limit of quarks, implicitly assuming that the $\mathrm{SU}(3)$ gauge modes are not critical [23,24]. We compare the renormalization-group (RG) predictions of the abovementioned field-theory approaches with numerical lattice results. This Letter allows us to deepen our understanding of their effectiveness and limitations, in particular for the widely used LGW approach.

To investigate the above issues, we consider lattice scalar gauge theories obtained by partially gauging a maximally symmetric model of matrix variables $Z_{x}^{a f}$. We start from the lattice action [25]

$$
S_{s}=-J \sum_{x, \mu} \operatorname{Re} \operatorname{Tr} Z_{x}^{\dagger} Z_{x+\hat{\mu}}, \quad \operatorname{Tr} Z_{x}^{\dagger} Z_{x}=1,
$$

where $Z_{x}^{a f}$ are $N_{c} \times N_{f}$ complex matrices and the sum is over all links of a cubic lattice ( $\hat{\mu}$ are unit vectors along the three lattice directions). The model has a global $\mathrm{O}(N)$ symmetry with $N=2 N_{c} N_{f}$. In particular, it is invariant under the global $\mathrm{SU}\left(N_{c}\right)$ transformations $Z_{x} \rightarrow V Z_{x}$, $V \in \mathrm{SU}\left(N_{c}\right)$. To make this symmetry a local one, we use the Wilson approach [21]. We associate an $\mathrm{SU}\left(N_{c}\right)$ matrix $U_{x, \hat{\mu}}$ with each link, and consider the action

$$
\begin{aligned}
S_{g}= & -\beta N_{f} \sum_{\boldsymbol{x}, \mu} \operatorname{Re} \operatorname{Tr}\left[Z_{\boldsymbol{x}}^{\dagger} U_{\boldsymbol{x}, \hat{\mu}} Z_{\boldsymbol{x}+\hat{\mu}}\right] \\
& -\frac{\beta_{g}}{N_{c}} \sum_{\boldsymbol{x}, \mu>\nu} \operatorname{Re} \operatorname{Tr}\left[U_{\boldsymbol{x}, \hat{\mu}} U_{\boldsymbol{x}+\hat{\mu}, \hat{\nu}} U_{\boldsymbol{x}+\hat{\nu}, \hat{\mu}}^{\dagger} U_{\boldsymbol{x}, \hat{\nu}}^{\dagger}\right],
\end{aligned}
$$

where the second sum is over all lattice plaquettes. Beside the $\mathrm{SU}\left(N_{c}\right)$ gauge invariance, the model also has a global $\mathrm{U}\left(N_{f}\right)$ symmetry, $Z_{x} \rightarrow Z_{x} U$ with $U \in \mathrm{U}\left(N_{f}\right)$. For $N_{c}=2$, the global symmetry group is larger than $\mathrm{U}\left(N_{f}\right)$. Indeed the action turns out to be invariant under the unitary symplectic group $\operatorname{Sp}\left(N_{f}\right) \supset \mathrm{U}\left(N_{f}\right)$ (see also Refs. [26,27]). If one defines the $2 \times 2 N_{f}$ matrix $\Gamma^{a l}$ as $\Gamma^{a f}=Z^{a f}, \Gamma^{a\left(N_{f}+f\right)}=$ $\sum_{b} \epsilon^{a b} \bar{Z}^{b f}\left(\epsilon^{a b}=-\epsilon^{b a}, \epsilon^{12}=1\right)$, one can show that the action is invariant under $\Gamma^{a l} \rightarrow \sum_{m} W^{l m} \Gamma^{a m}$, where

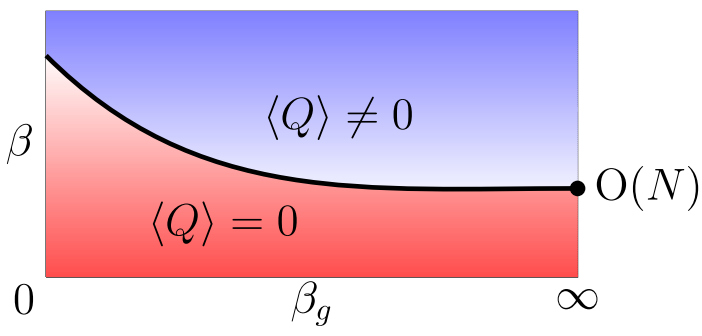

FIG. 1. Sketch of the phase diagram of 3D lattice scalar chromodynamics with $N_{f} \geq 2$. The transition line is continuous for $N_{f}=2$ and of first order for $N_{f} \geq 3$. We conjecture that its nature is the same for any finite $\beta_{g}$. The endpoint for $\beta_{g} \rightarrow \infty$ is the $\mathrm{O}(N)$ critical point $\left(N=2 N_{c} N_{f}\right)$.

$W \in \operatorname{Sp}\left(N_{f}\right)$, and $l, m=1, \ldots, 2 N_{f}$. For $\beta_{g}=\infty$, the gauge fields are equal to the identity (modulo gauge transformations); thus we recover the ungauged model in Eq. (1), i.e., the standard $N$ vector model with $N=2 N_{c} N_{f}$.

For $N_{f}=1$, no transition [28] is expected for finite $\beta_{g}$ [29-31] (we have verified it numerically up to $\beta_{g}=6$ ), and long-range correlations should only develop for $\beta_{g} \rightarrow \infty$ close to the $\mathrm{O}\left(2 N_{c}\right)$ critical point. For $N_{f} \geq 2$, we find a transition line that separates two different phases-see Fig. 1-which are characterized by the behavior of the gauge-invariant order parameter

$$
Q_{x}^{f g}=\sum_{a} \bar{Z}_{x}^{a f} Z_{x}^{a g}-\frac{1}{N_{f}} \delta^{f g},
$$

which is a Hermitian and traceless $N_{f} \times N_{f}$ matrix. The nature of the transition depends on $N_{f}$ and $N_{c}$, while it does not depend on the gauge coupling $\beta_{g}$.

Before presenting the numerical results, we discuss the predictions of the statistical field theories that may describe the behavior along the transition line. We begin by considering the continuum scalar chromodynamics defined by the Lagrangian

$$
\mathcal{L}=\frac{1}{4 g^{2}} \operatorname{Tr} F_{\mu \nu}^{2}+\operatorname{Tr}\left[\left(D_{\mu} Z\right)^{\dagger}\left(D_{\mu} Z\right)\right]+V\left(\operatorname{Tr} Z^{\dagger} Z\right),
$$

where $V(X)=r X+\frac{1}{6} u X^{2}, F_{\mu \nu}=\partial_{\mu} A_{\nu}-\partial_{\nu} A_{\mu}+\left[A_{\mu}, A_{\nu}\right]$, and $D_{\mu, a b}=\partial_{\mu} \delta_{a b}+t_{a b}^{c} A_{\mu}^{c}$. The RG flow in the space of the renormalized couplings $u$ and $f \equiv g^{2}$ can be studied perturbatively within the $\varepsilon \equiv 4-D$ expansion [32]. At one loop, the $\beta$ functions read [33-37]

$$
\begin{gathered}
\beta_{f}(u, f) \equiv \mu \frac{\partial f}{\partial \mu}=-\varepsilon f-\left(22 N_{c}-N_{f}\right) f^{2} \\
\beta_{u}(u, f) \equiv \mu \frac{\partial u}{\partial \mu}=-\varepsilon u+\left(N_{f} N_{c}+4\right) u^{2}-\frac{18\left(N_{c}^{2}-1\right)}{N_{c}} u f \\
+\frac{27\left(N_{c}-1\right)\left(N_{c}^{2}+2 N_{c}-2\right)}{N_{c}^{2}} f^{2} .
\end{gathered}
$$


A stable fixed point (FP) is only found for $N_{f}>N_{f}^{*}\left(N_{c}\right)$ with $N_{f}^{*}(2)=359+O(\varepsilon)$ and $N_{f}^{*}(3)=972.95+O(\varepsilon)$. Therefore, for any $N_{f}<N_{f}^{*}\left(N_{c}\right)$, and, in particular, for small values of $N_{f}$, the transition is predicted to be first order. We also note that for large $\beta_{g}$ the lattice model (2) is expected to show significant crossover effects due to the nearby $\mathrm{O}(N)$ transition point $\left(N=2 N_{c} N_{f}\right)$. In the theory (4) such a crossover is controlled by the RG flow in the vicinity of the $\mathrm{O}(N)$ fixed point $\left[f^{*}=0\right.$ and $u_{N}^{*}=\varepsilon$ / $\left.\left(N_{f} N_{c}+4\right)\right]$, which is always unstable with respect to the gauge perturbation [38].

An alternative field-theoretical approach is provided by the LGW framework [15,32,39-42], in which one assumes that the critical modes are associated with a gauge-invariant composite operator. In the present case, the natural order parameter is the operator $Q_{x}^{f g}$ defined in Eq. (3). This is a nontrivial assumption, as it postulates that gauge fields do not play a relevant role in the effective theory of the critical modes. The LGW fundamental field is correspondingly a traceless Hermitian matrix $\Psi^{f g}(\boldsymbol{x})$, which can be formally considered as the average of $Q_{x}^{f g}$ over a large but finite domain. The LGW theory is obtained by considering the most general fourth-order polynomial consistent with the global symmetry

$$
\operatorname{Tr}\left(\partial_{\mu} \Psi\right)^{2}+r \operatorname{Tr} \Psi^{2}+w \operatorname{tr} \Psi^{3}+u\left(\operatorname{Tr} \Psi^{2}\right)^{2}+v \operatorname{Tr} \Psi^{4} .
$$

Continuous transitions may only occur if its RG flow has a stable FP. For $N_{f}=2$, the cubic term vanishes and the two quartic terms are equivalent, leading to the $\mathrm{O}(3)$-symmetric LGW theory. This implies that the phase transition can be continuous, in the $\mathrm{O}(3)$ universality class because of the mapping $\mathrm{SO}(3)=\mathrm{SU}(2) / \mathbb{Z}_{2}$. An explicit $\mathrm{O}(3)$ order parameter is obtained by considering the real vector variable $\varphi_{x}^{k}=\sum_{f g} \sigma_{f g}^{k} Q_{x}^{f g}$, where $\sigma^{k}$ are the Pauli matrices. For $N_{f} \geq 3$, the cubic $\Psi^{3}$ term is generically expected to be present. For 3D systems, this is usually taken as an indication that phase transitions are generically first order [43].

The above arguments apply to generic $N_{c}>2$. Since for $N_{c}=2$ the global symmetry is $\operatorname{Sp}\left(N_{f}\right)$, the order parameter is now a $2 N_{f} \times 2 N_{f}$ matrix given by

$$
\mathcal{T}_{\boldsymbol{x}}^{l m}=\sum_{a} \bar{\Gamma}_{x}^{a l} \Gamma_{x}^{a m}-\frac{\delta^{l m}}{2 N_{f}} \sum_{a n} \bar{\Gamma}_{x}^{a n} \Gamma_{\boldsymbol{x}}^{a n},
$$

which can be expressed in terms of $Q_{x}^{f g}$ defined in Eq. (3) $\left(\mathcal{T}_{x}^{f g}=Q_{x}^{f g}\right.$ for $\left.f, g=1, \ldots, N_{f}\right)$ and of $D_{x}^{f g}=$ $\sum_{a b} \epsilon^{a b} Z_{x}^{a f} Z_{x}^{b g}$. For $N_{f}=2$, the $\operatorname{Sp}(2)$ group is isomorphic to the $\mathrm{O}(5)$ group. The $\mathrm{Sp}(2) \mathrm{LGW}$ theory is that of an $\mathrm{O}(5)$-symmetric vector model. Indeed, the order parameter can be rewritten in terms of the three-component vector $\varphi_{x}^{k}=\sum_{f g} \sigma_{f g}^{k} Q_{x}^{f g}$ and the real and imaginary parts of the complex variable $\phi_{x}=\frac{1}{2} \sum_{f g} \epsilon^{f g} D_{x}^{f g}$. They form a fivecomponent order parameter. Thus we predict an $\mathrm{O}(5)$ critical behavior. For larger $N_{f}$, the $\operatorname{Sp}\left(N_{f}\right)$ LGW theory contains cubic interactions [44], and therefore first-order transitions are predicted.

Summarizing, the LGW approach based on a gaugeinvariant order parameter predicts that continuous transitions only occur for $N_{f}=2$. They belong to the $\mathrm{O}(3)$ universality class for any $N_{c} \geq 3$ and to the $\mathrm{O}(5)$ universality class for $N_{c}=2$. Instead, first-order transitions are generically expected for $N_{f} \geq 3$ and any $N_{c}$. Note that for $N_{f}=2$ the LGW predictions differ from those of the continuum gauge theory (4), as the latter predicts a firstorder transition (no FP for $N_{f}=2$ ). The two theories apparently also disagree for large values of $N_{f}$. The continuum gauge theory admits the possibility of continuous transitions, since a stable FP exists, while the LGW theory indicates first-order transitions for any $N_{f}>2$.

In our numerical study [45], we consider the model (2) on a cubic lattice of size $L$ and periodic boundary conditions. We compute the correlation $G(\boldsymbol{x}-\boldsymbol{y})=\left\langle\operatorname{Tr} Q_{x} Q_{y}\right\rangle$ of the composite operator $Q_{x}$ defined in Eq. (3), its susceptibility $\chi=\sum_{x} G(\boldsymbol{x})$, and correlation length $\xi$,

$$
\xi^{2} \equiv \frac{1}{4 \sin ^{2}(\pi / L)} \frac{\tilde{G}(\mathbf{0})-\tilde{G}\left(\boldsymbol{p}_{m}\right)}{\tilde{G}\left(\boldsymbol{p}_{m}\right)},
$$

where $\tilde{G}(\boldsymbol{p})=\sum_{x} e^{i \boldsymbol{p} \cdot \boldsymbol{x}} G(\boldsymbol{x})$ and $\boldsymbol{p}_{m}=(2 \pi / L, 0,0)$. We also consider the Binder parameter

$$
U=\frac{\left\langle\mu_{2}^{2}\right\rangle}{\left\langle\mu_{2}\right\rangle^{2}}, \quad \mu_{2}=\frac{1}{L^{6}} \sum_{x, y} \operatorname{Tr} Q_{x} Q_{y} .
$$

At continuous transitions, RG-invariant quantities, such as $R_{\xi} \equiv \xi / L$ and $U$, behave as $[22,42]$

$R(\beta, L)=f_{R}(X)+O\left(L^{-\omega}\right), \quad X=\left(\beta-\beta_{c}\right) L^{1 / \nu}$,

where $\nu$ is the correlation-length exponent, $f_{R}(X)$ is a universal function (apart from a normalization of the argument), and $\omega$ is the exponent associated with the leading scaling corrections. Moreover, since $R_{\xi}$ is a monotonic function, Eq. (11) implies $U(\beta, L) \approx F_{U}\left(R_{\xi}\right)$, where $F_{U}$ depends on the universality class only, without free normalizations (once fixed the boundary conditions and the shape of the lattice).

The results for $N_{f}=2, N_{c}=3$, and $\beta_{g}=0$ up to $L=$ 64 (see Fig. 2) confirm that the transition at $\beta_{c}=3.7518(2)$ is continuous and belongs to the $3 \mathrm{D} \mathrm{O}(3)$ universality class, characterized by the universal exponents $[42,46-48]$ $\nu=0.7117(5), \eta=0.0378(3)$, and $\omega=0.782(13)$. We obtained analogous results for $\beta_{g}=3\left[\beta_{c}=3.203(1)\right]$ and for $N_{c}=4$ at $\beta_{g}=0\left[\beta_{c}=4.896(1)\right]$ (see Fig. 3) supporting the $\mathrm{O}(3)$ nature of the transition in both cases. 


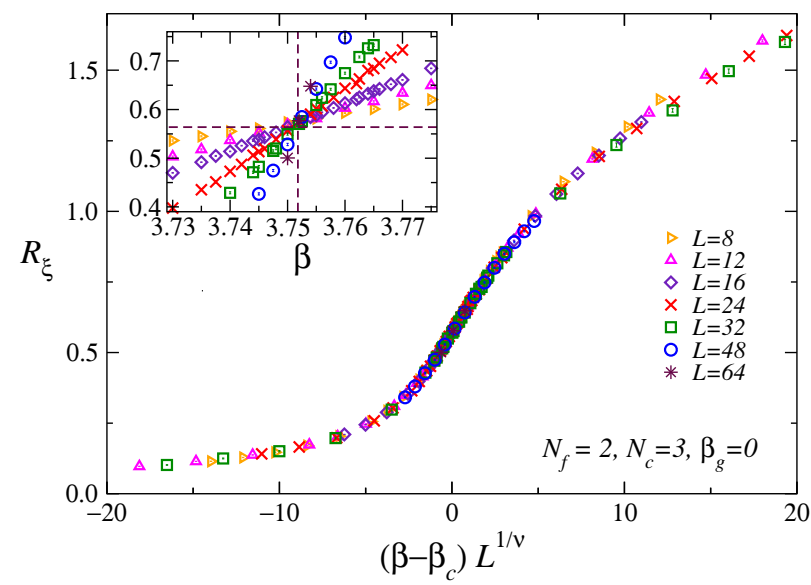

FIG. 2. $\quad R_{\xi}$ vs $\left(\beta-\beta_{c}\right) L^{1 / \nu}$ for $N_{f}=2, N_{c}=3$, and $\beta_{g}=0$, up to $L=64$. We use the $\mathrm{O}(3)$ value $[46,47] \nu=0.7117(5)$ and $\beta_{c}=3.7518(2)$. The data collapse on a unique curve confirms the $\mathrm{O}$ (3) critical behavior. (Inset) $R_{\xi}$ vs $\beta$. The vertical dashed line corresponds to $\beta_{c}$, while the horizontal one corresponds to the critical value $R_{\xi}^{*}$, which is consistent with the $\mathrm{O}(3)$ value $R_{\xi}^{*}=0.5639(2)$.

These results confirm the LGW predictions. They lead us to conjecture that the phase diagram for $N_{f}=2$ and $N_{c} \geq 3$ is characterized by a continuous transition line, related to the condensation of the order parameter $Q_{x}$, which belongs to the $\mathrm{O}(3)$ universality class for any finite $\beta_{g}$. For $\beta_{g} \rightarrow \infty$, the critical behavior turns into that of the $\mathrm{O}(N)$ vector model with $N=4 N_{c}$.

We have also performed a finite size scaling analysis for $N_{f}=N_{c}=2$ at $\beta_{g}=0$ (up to $L=96$ ) and $\beta_{g}=2$ (up to

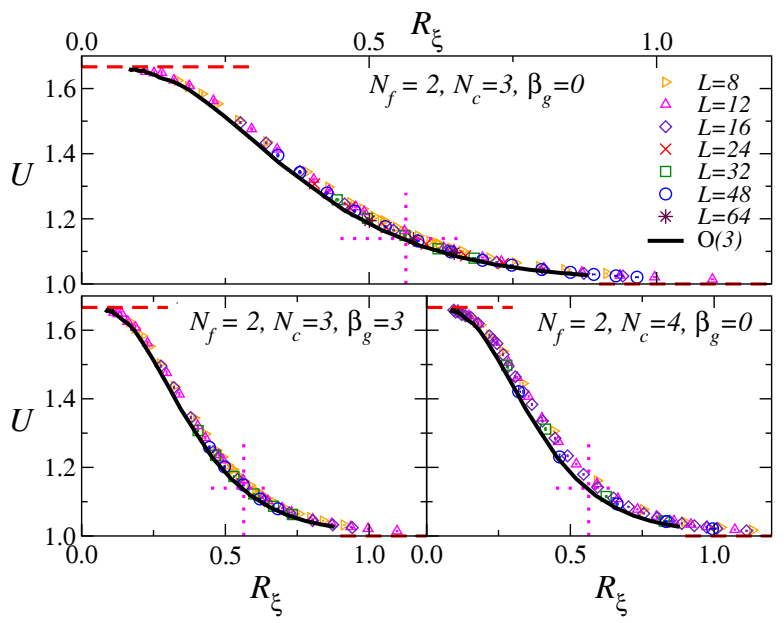

FIG. 3. Binder parameter $U$ vs $R_{\xi}$ for $N_{f}=2$ models: results for $N_{c}=3, \beta_{g}=0$ (top), for $N_{c}=3, \beta_{g}=3$ (bottom left), and for $N_{c}=4, \beta_{g}=0$ (bottom right). In all cases, the data appear to converge to the $\mathrm{O}(3)$ scaling curve, obtained by Monte Carlo simulations of the $\mathrm{O}(3)$ vector model [44]. The dotted horizontal and vertical lines correspond to the $\mathrm{O}(3)$ critical values [46] $U^{*}=$ $1.1394(3)$ and $R_{\xi}^{*}=0.5639(2)$.
$L=64)$. In both cases, we observe continuous transitions, at $\beta_{c}=2.68885(5)$ and $\beta_{c}=1.767(1)$, respectively [note that $\beta_{c}=0.96339(1)$ in the $\mathrm{O}(8)$ vector model [49] obtained for $\left.\beta_{g} \rightarrow \infty\right]$. Data are consistent with the $\mathrm{O}(5)$ universality class, whose critical exponents are [50-53] $\nu=0.779(3), \eta=0.034(1)$, and $\omega=0.79(2)$. Numerical results [44] for $\beta_{g}=0$ are shown in Fig. 4. They confirm the predictions of the LGW theory based on the enlarged global symmetry group $\mathrm{Sp}(2) \approx \mathrm{O}(5)$. We note that the enlarged $\mathrm{O}(5)$ symmetry may be seen as emerging from the combination of an $\mathrm{O}(3)$ "magneticlike" and of a $\mathrm{U}(1)$ "superfluidlike" order parameter [5,6,44]. Our results characterize the critical behavior in the presence of emergent $\mathrm{O}(5)$ symmetries [5,6,54-60].

Finally, we mention that we also studied models for $N_{f}=3$ and $N_{c}=2$ and 3 , at $\beta_{g}=0$. The numerical results provide evidence of a first-order transition in both cases [44]. This is again consistent with the predictions of the effective LGW theory (7).

In conclusion, we have investigated the phase diagram of the lattice multiflavor scalar chromodynamics (2), for positive couplings $\beta$ and $\beta_{g}$. This is a paradigmatic 3D model with a non-Abelian gauge symmetry. For $N_{f} \geq 2$, the phase diagram is characterized by two phases: a lowtemperature phase in which the order parameter $Q_{x}^{f g}$ condenses and a high-temperature disordered phase where it vanishes. Gauge and vector observables do not show long-range correlations for any finite $\beta$ and $\beta_{g}$. The two phases are separated by a transition line driven by the condensation of $Q_{x}^{f g}$, as sketched in Fig. 1, that ends at the unstable $\mathrm{O}(N)$ transition point with $N=2 N_{c} N_{f}$ for $\beta_{g} \rightarrow \infty$. The gauge coupling $\beta_{g}$ does not play any

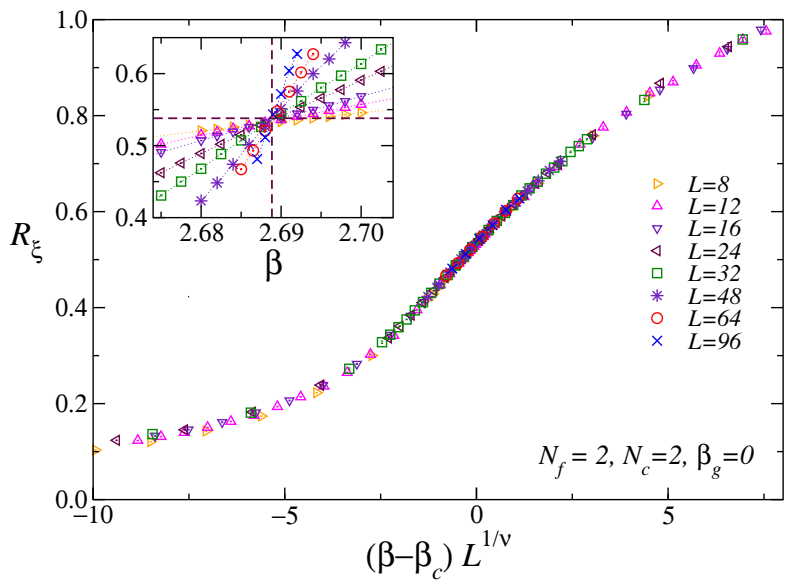

FIG. 4. $\quad R_{\xi}$ vs $\left(\beta-\beta_{c}\right) L^{1 / \nu}$ for $N_{f}=2, N_{c}=2$, and $\beta_{g}=0$, up to $L=96$. We use the $\mathrm{O}(5)$ value [51] $\nu=0.779(3)$ and $\beta_{c}=2.68885(5)$. The data collapse supports the $\mathrm{O}(5)$ critical behavior. (Inset) Data vs $\beta$ : at $\beta_{c}$ (vertical dashed line), $R_{\xi}$ is consistent with the $\mathrm{O}(5)$ value $R_{\xi}^{*}=0.538(1)$ (horizontal dashed line). Plots of $U$ vs $R_{\xi}$ as in Fig. 3 also support the $\mathrm{O}(5)$ critical behavior. 
particular role: the nature of the transition is conjectured to be the same for any $\beta_{g}$, as numerically checked for some values of $\beta_{g}$. Along the transition line, only correlations of the gauge-invariant operator $Q_{x}^{a b}$ display long-range order. Gauge modes are not critical and only represent a background that gives rise to crossover effects.

The numerical results are compared with the predictions of the continuum scalar gauge theory (2) and of the gaugeinvariant LGW theory (7). They agree with those of the LGW theory, showing that the LGW framework provides the correct description of the large-scale behavior of these systems along the transition line separating the low- and high-temperature phases, predicting first-order transitions for $N_{f}=3$ and continuous transitions for $N_{f}=2$, which belong to the $\mathrm{O}(3)$ universality class for $N_{c} \geq 3$ and the $\mathrm{O}(5)$ universality class for $N_{c}=2$. On the other hand, the results for $N_{f}=2$ are in contradiction with the predictions of the continuum gauge model (2): as no stable FP exists for $N_{f}=2$, one would expect a first-order transition. There are at least two possible explanations for this apparent failure. A first possibility is that it does not encode the relevant modes at the transition. A second possibility is that the $\varepsilon$-expansion perturbative treatment around $4 \mathrm{D}$, based on Eqs. (5) and (6), does not provide the correct description of the 3D behavior [44]. The 3D FP may not be related to a 4D FP, and therefore it escapes any perturbative analysis in powers of $\varepsilon \equiv 4-D$. The analysis of the behavior in the large- $N_{f}$ limit, where again the two approaches give different results, may help to shed light on these issues. Similar issues for the multicomponent lattice scalar electrodynamics are addressed in Refs. $[15,61]$.

We have considered a paradigmatic lattice model obtained by gauging a maximally symmetric scalar system. It would be interesting to consider scalar theories with different global and local symmetries and different symmetry-breaking patterns. Their classification deserves further investigation.

The LGW approach based on a gauge-invariant order parameter can be extended to systems with fermionic fields as well. It has been applied to the finite-temperature transition of quantum chromodynamics (QCD) with fermionic matter [23,24]. The lattice-QCD numerical results have only partially confirmed the LGW predictions, due to the complexity of the simulations with fermions [62]. Our results support the effectiveness of the LGW approach, since the derivation of the LGW theory is essentially independent of the bosonic or fermionic nature of the matter fields. Our results may be particularly useful to predict the symmetrybreaking patterns and critical behaviors at thermal and quantum phase transitions in condensed-matter systems with emerging non-Abelian gauge fields $[2,5,6]$.

Numerical simulations have been performed on the CSN4 cluster of the Scientific Computing Center at INFN-PISA.
[1] S. Weinberg, The Quantum Theory of Fields (Cambridge University Press, Cambridge, England, 2005).

[2] S. Sachdev, Topological order, emergent gauge fields, and Fermi surface reconstruction, Rep. Prog. Phys. 82, 014001 (2019).

[3] P. W. Anderson, Plasmons, gauge Invariance, and mass, Phys. Rev. 130, 439 (1963); Superconductivity: Higgs, Anderson and all that, Nat. Phys. 11, 93 (2015).

[4] T. Senthil, L. Balents, S. Sachdev, A. Vishwanath, and M. P. A. Fisher, Quantum criticality beyond the LandauGinzburg-Wilson paradigm, Phys. Rev. B 70, 144407 (2004).

[5] C. Wang, A. Nahum, M. A. Metlitski, C. Xu, and T. Senthil, Deconfined Quantum Critical Points: Symmetries and Dualities, Phys. Rev. X 7, 031051 (2017).

[6] S. Gazit, F. F. Assaad, S. Sachdev, A. Vishwanath, and C. Wang, Confinement transition of $Z_{2}$ gauge theories coupled to massless fermions: Emergent $\mathrm{QCD}_{3}$ and SO(5) symmetry, Proc. Natl. Acad. Sci. U.S.A. 115, E6987 (2018).

[7] S. Sachdev, H. D. Scammell, M. S. Scheurer, and G. Tarnopolsky, Gauge theory for the cuprates near optimal doping, Phys. Rev. B 99, 054516 (2019).

[8] H. Goldman, R. Sohal, and E. Fradkin, LandauGinzburg Theories of non-Abelian quantum Hall states from non-Abelian bosonization, Phys. Rev. B 100, 115111 (2019).

[9] D. Boyanovsky, H. J. de Vega, and D. J. Schwarz, Phase transitions in the early and present universe, Annu. Rev. Nucl. Part. Sci. 56, 441 (2006).

[10] J. Adams et al. (STAR Collaboration), Experimental and theoretical challenges in the search for the quark gluon plasma: The STAR collaboration's critical assessment of the evidence from RHIC collisions, Nucl. Phys. A757, 102 (2005).

[11] F. Englert and R. Brout, Broken Symmetry and the Mass of Gauge Vector Mesons, Phys. Rev. Lett. 13, 321 (1964); P. W. Higgs, Broken Symmetries and the Masses of Gauge Bosons, Phys. Rev. Lett. 13, 508 (1964); G. S. Guralnik, C. R. Hagen, and T. W. B. Kibble, Global Conservation Laws and Massless Particles, Phys. Rev. Lett. 13, 585 (1964).

[12] Y.-Z. You, Y.-C. He, C. Xu, and A. Vishwanath, Symmetric Fermion Mass Generation as Deconfined Quantum Criticality, Phys. Rev. X 8, 011026 (2018).

[13] Y.-Z. You, Y.-C. He, A. Vishwanath, and C. Xu, From bosonic topological transition to symmetric fermion mass generation, Phys. Rev. B 97, 125112 (2018).

[14] A. Thomson and S. Sachdev, Fermionic Spinon Theory of Square Lattice Spin Liquids near the Néel State, Phys. Rev. X 8, 011012 (2018).

[15] A. Pelissetto and E. Vicari, Multicomponent compact abelian Higgs models, Phys. Rev. E 100, 042134 (2019); Three-dimensional ferromagnetic $\mathrm{CP}^{N-1}$ models, Phys. Rev. E 100, 022122 (2019).

[16] S. Nadkarni, The SU(2) Aajoint Higgs model in threedimensions, Nucl. Phys. B334, 559 (1990).

[17] K. Kajantie, K. Rummukainen, and M. E. Shaposhnikov, A lattice Monte Carlo study of the hot electroweak phase transition, Nucl. Phys. B407, 356 (1993). 
[18] W. Buchmuller and O. Philipsen, Phase structure and phase transition of the SU(2) Higgs model in three-dimensions, Nucl. Phys. B443, 47 (1995).

[19] K. Kajantie, M. Laine, K. Rummukainen, and M. E. Shaposhnikov, Is There a Hot Electroweak Phase Transition at $\mathrm{m}(\mathrm{H})$ Larger or Equal to m(W)?, Phys. Rev. Lett. 77, 2887 (1996).

[20] A. Hart, O. Philipsen, J. D. Stack, and M. Teper, On the phase diagram of the $S U(2)$ adjoint Higgs model in $(2+1)$ dimensions, Phys. Lett. B 396, 217 (1997).

[21] K. G. Wilson, Confinement of quarks, Phys. Rev. D 10, 2445 (1974).

[22] J. Zinn-Justin, Quantum Field Theory and Critical Phenomena (Oxford University Press, Oxford, 2002).

[23] R. D. Pisarski and F. Wilczek, Remarks on the chiral phase transition in chromodynamics, Phys. Rev. D 29, 338 (1984).

[24] A. Pelissetto and E. Vicari, Relevance of the axial anomaly at the finite-temperature chiral transition in QCD, Phys. Rev. D 88, 105018 (2013); A. Butti, A. Pelissetto, and E. Vicari, On the nature of the finite-temperature transition in QCD, J. High Energy Phys. 08 (2003) 029.

[25] Note that the unit-length constraint of the $Z_{x}$ variables can be considered as a particular limit of a quartic potential term $\sum_{x} V\left(\operatorname{Tr} Z_{x}^{\dagger} Z_{x}\right)$ with $V(X)=r X+\frac{1}{6} u X^{2}$.

[26] H. Georgi, Weak Interactions and Modern Particle Theory (Benjamin/Cummings Publishing Company, Menlo Park, 1984).

[27] P. S. Bhupal Dev and A. Pilaftsis, Maximally symmetric two Higgs doublet model with natural standard model alignment, J. High Energy Phys. 12 (2014) 024; Erratum, J. High Energy Phys. 11 (2015) 147.

[28] For $N_{f}=1$ the unit-length condition of the $Z$ variables, cf. Eq. (1), allows us to get rid of them by a gauge transformation, hence no transition is possible.

[29] K. Osterwalder and E. Seiler, Gauge field theories on the lattice, Ann. Phys. (N.Y.) 110, 440 (1978).

[30] E. Fradkin and S. Shenker, Phase diagrams of lattice gauge theories with Higgs fields, Phys. Rev. D 19, 3682 (1979).

[31] S. Dimopoulos, S. Raby, and L. Susskind, Light composite fermions, Nucl. Phys. B173, 208 (1980).

[32] K. G. Wilson and J. Kogut, The renormalization group and the $\epsilon$ expansion, Phys. Rep. 12, 75 (1974).

[33] D. J. Gross and F. Wilczek, Ultraviolet Behavior of NonAbelian Gauge Theories, Phys. Rev. Lett. 30, 1343 (1973).

[34] T. P. Cheng, E. Eichten, and L.-F. Li, Higgs phenomena in asymptotically free gauge theories, Phys. Rev. D 9, 2259 (1974).

[35] P. Arnold and L. G. Yaffe, The $\epsilon$ expansion and the electroweak phase transition, Phys. Rev. D 49, 3003 (1994).

[36] A. Das, Phase transition in $\mathrm{SU}(N) \times \mathrm{U}(1)$ gauge theory with many fundamental bosons, Phys. Rev. B 97, 214429 (2018).

[37] We rescale the couplings $u$ and $f$ as $u \rightarrow u /\left(24 \pi^{2}\right)$ and $f \rightarrow f /\left(48 \pi^{2}\right)$ to simplify the expressions.

[38] The corresponding eigenvalue of the stability matrix is negative, $\lambda_{f}=-\varepsilon+O\left(\varepsilon^{2}\right)$ for any $N_{f}$ and $N_{c}$.

[39] L. D. Landau and E. M. Lifshitz, Statistical Physics. Part I, 3rd ed. (Elsevier Butterworth-Heinemann, Oxford, 1980).
[40] M.E. Fisher, The renormalization group in the theory of critical behavior, Rev. Mod. Phys. 47, 543 (1975).

[41] S.-k. Ma, Modern Theory of Critical Phenomena (W.A. Benjamin, Reading, MA, 1976).

[42] A. Pelissetto and E. Vicari, Critical phenomena and renormalization group theory, Phys. Rep. 368, 549 (2002).

[43] The presence of a cubic term in LGW theory is usually taken as an indication that the phase transitions occurring in the corresponding class of systems are first order. Indeed, a straightforward mean-field analysis shows that the transition is first order in four dimensions. The nature of the transition should not change sufficiently close to four dimensions, as long as statistical fluctuations are small. In particular, it is usually assumed that the 4D mean-field predictions also apply in three dimensions.

[44] A more detailed report will be presented elsewhere, C. Bonati, A. Pelissetto, and E. Vicari (to be published).

[45] We perform Monte Carlo simulations using an overrelaxed algorithm that consists in a mixture of microcanonical, heatbath, and Metropolis updates.

[46] M. Campostrini, M. Hasenbusch, A. Pelissetto, P. Rossi, and E. Vicari, Critical exponents and equation of state of the three-dimensional Heisenberg universality class, Phys. Rev. B 65, 144520 (2002).

[47] M. Hasenbusch and E. Vicari, Anisotropic perturbations in 3D $\mathrm{O}(N)$ vector models, Phys. Rev. B 84, 125136 (2011).

[48] R. Guida and J. Zinn-Justin, Critical exponents of $N$-vector models, J. Phys. A 31, 8103 (1998).

[49] F. Delfino, A. Pelissetto, and E. Vicari, Three-dimensional antiferromagnetic $C P^{N-1}$ models, Phys. Rev. E 91, 052109 (2015).

[50] S. A. Antonenko and A. I. Sokolov, Critical exponents for a three-dimensional $\mathrm{O}(n)$-symmetric model with $n>3$, Phys. Rev. E 51, 1894 (1995).

[51] M. Hasenbusch, A. Pelissetto, and E. Vicari, Instability of the $\mathrm{O}(5)$ critical behavior in the $\mathrm{SO}(5)$ theory of high- $T_{c}$ superconductors, Phys. Rev. B 72, 014532 (2005).

[52] L. A. Fernández, V. Martín-Mayor, D. Sciretti, A. Tarancón, and J. L. Velasco, Numerical study of the enlarged $\mathrm{O}(5)$ symmetry of the 3-D antiferromagnetic $\mathrm{RP}^{2}$ spin model, Phys. Lett. B 628, 281 (2005).

[53] P. Calabrese, A. Pelissetto, and E. Vicari, Multicritical behavior of $\mathrm{O}\left(n_{1}\right) \oplus \mathrm{O}\left(n_{2}\right)$-symmetric systems, Phys. Rev. B 67, 054505 (2003).

[54] A. Nahum, P. Serna, J. T. Chalker, M. Ortun, and A. M. Somoz, Emergent $\mathrm{SO}(5)$ Symmetry at the Néel to Valence Bond Solid Transition, Phys. Rev. Lett. 115, 267203 (2015).

[55] G. J. Sreejith, S. Powell, and A. Nahum, Emergent SO(5) Symmetry at the Columnar Ordering Transition in the Classical Cubic Dimer Model, Phys. Rev. Lett. 122, 080601 (2019).

[56] Z.-X. Li, S.-K. Jian, and H. Yao, Deconfined quantum criticality and emergent $\mathrm{SO}(5)$ symmetry in fermionic systems arXiv:1904.10975. 
[57] F. Wu, I. Sodemann, Y. Araki, A. H. MacDonald, and T. Jolicoeur, $\mathrm{SO}(5)$ symmetry in the quantum Hall effect in graphene, Phys. Rev. B 90, 235432 (2014).

[58] C. Xu and S. Sachdev, A Square Lattice Algebraic Spin Liquid with SO(5) Symmetry, Phys. Rev. Lett. 100, 137201 (2008).

[59] X. Hu, T. Koyama, and M. Tachiki, Phase Diagram of a Superconducting and Antiferromagnetic System with SO(5) Symmetry, Phys. Rev. Lett. 82, 2568 (1999).
[60] C. L. Henley, Exact Realization of SO(5) Symmetry in Extended Hubbard Models, Phys. Rev. Lett. 80, 3590 (1998).

[61] Interestingly, 3D multicomponent Abelian Higgs models show phase diagrams, symmetry-breaking patterns, and critical behaviors analogous to those of non-Abelian scalar chromodynamics for $N_{c} \geq 3$.

[62] S. Sharma, Recent progress on the QCD phase diagram, Proc. Sci. LATTICE2018 (2019) 009. 